\title{
Recent observations of WD1524 at MIRO and participation in WET observing program
}

\author{
H. O. Vats \& K. S. Baliyan \\ Physical Research Laboratory, Ahmedabad 380009, INDIA
}

\begin{abstract}
Most stars end up as white dwarfs on their evolutionary track. A knowledge of the structure of white dwarfs puts constraints on their prior evolutionary stages. By conducting co-ordinated continuous long term observations of these pulsating objects, high precision light curves can be generated to study their environments. We report preliminary photometric observations of WD J1524 from Mt Abu Infrared Observatory (MIRO). The photometric data collected for this source during 27-28 May 2007, though not of sufficient duration, indicate a periodicity of $210 \mathrm{~s}$. These observations provide us confidence to join the future observing programs of Whole Earth Telescope (WET) for such studies.
\end{abstract}

\section{Introduction}

Asteroseismology via precise photometry provides a useful tool to probe the interiors of the pulsating stars (Winget 1998). The white dwarfs (WD) show pulsations, with a range of periodicity frequencies and several tens of millimag amplitude variations which makes them good candidates for the study of stellar evolution. Most of the stars in the universe end up as white dwarfs in their evolutionary phase, and their structures can be used to put constraints on their prior evolutionary stages. As they are faint objects only the nearby objects have been detected so far. However, a large number of white dwarfs should be present in the Galaxy. Determining the contribution of white dwarfs to the total mass of the Galaxy could help solve one of the fundamental questions in modern astronomy: presence and extent of dark matter. The properties of white dwarfs, high gravity and temperature, are important means for the study of physics under extreme conditions. Therefore a sample of white dwarfs are 


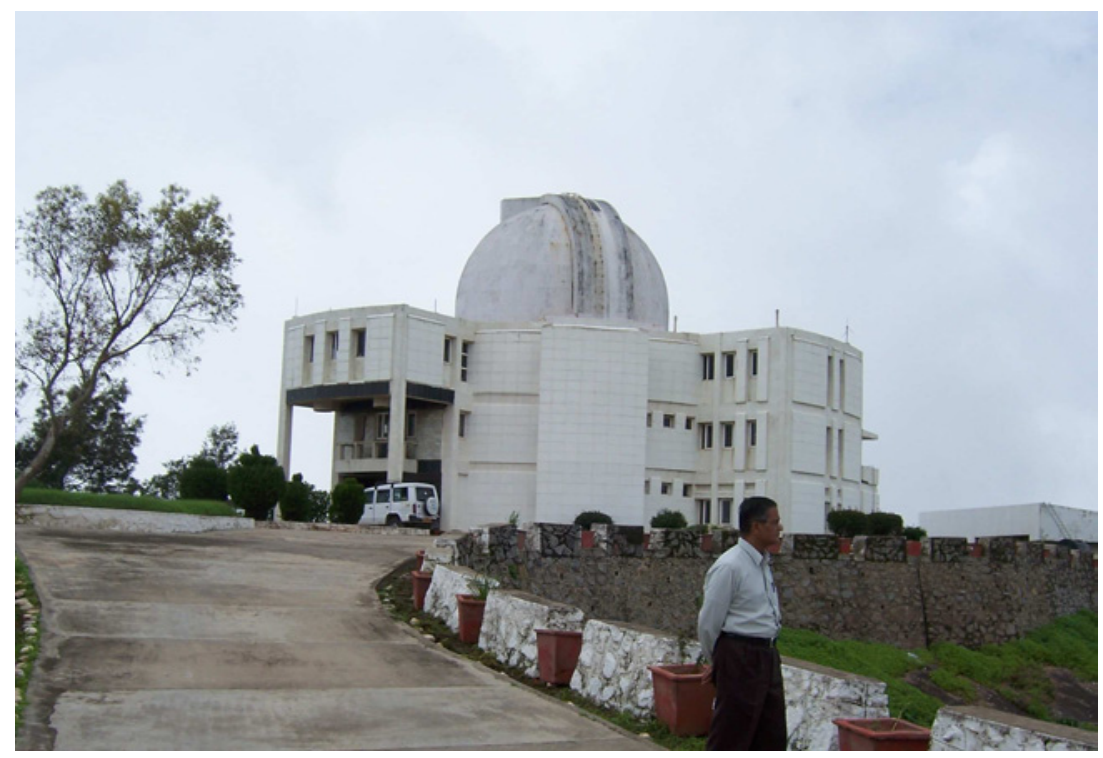

Figure 1: A view of the Mt. Abu Infrared Observatory (MIRO).

being observed in the Whole Earth Telescope campaign (Mukadam et al. 2006) to get as precise and complete a light curve as possible.

\section{Observations}

In an attempt to study the feasibility and exploratory aspects of our joining the WET campaign, optical photometry observations of white dwarf WD1524 were carried out from the Mt Abu Infrared Observatory, Mt Abu, Rajasthan, India.

\section{Mt. Abu Observatory}

Mt Abu Infrared Observatory (MIRO) is on top of the Gurushikhar peak (Longitude: 72:46:47 E, Latitude: 24:39:10 N) of Aravali range in Rajasthan state of Western India. It houses a $1.2 \mathrm{~m}$ Cassegrain focus $\mathrm{f} / 13$ telescope, optimized for infrared and optical observations (Deshpande 1995). The Observatory is situated near the highest peak in Aravali range of mountains at $1680 \mathrm{~m}$ height above the mean sea level. A view of the observatory is shown in Figure 1.

The Mt. Abu telescope is an equatorially mounted open truss and fork type and has a $1.2 \mathrm{~m}$ parabolic primary $(\mathrm{f} / 3)$ and $300 \mathrm{~mm}$ hyperbolic secondary. It has a Cassegrain focus $(f / 13)$ behind the primary at a nominal 
distance of $380 \mathrm{~mm}$ from the Cassegrain plate/instrument ring (Deshpande 1995). At $15.6 \mathrm{~m}$ effective focal length of the telescope, the plate scale is $13 " / \mathrm{mm}$ that remains linear over the telescope field of view of 10 arcmin diameter. The diameter of the primary central hole is $230 \mathrm{~mm}$. The primary was polished from a mirror-blank of Cervit and weighs $300 \mathrm{~kg}$ whereas the secondary made up of Zerodur weighs $6 \mathrm{~kg}$. The primary mirror is mounted in a mirror cell enclosure and is supported on floating axial (18 nos.) and radial (12 nos.) support pads. Each supporting pad is connected to an astatic lever and a balancing counterweight. The mirror support system allows the mirror to float inside the mirror cell with maximum deformation of $20 \mathrm{~nm}$ over the entire mirror surface against the cell flexure in any telescope position. The secondary mirror is mounted with a mounting ring at a distance of $2820 \mathrm{~mm}$ in front of the primary and is supported by 4 streamlined radial wings. For mounting a back-end instrument on the telescope, an instrument ring of $450 \mathrm{~mm}$ diameter is provided. The instrument ring can be rotated about the optical axis of the telescope as it is attached to a bearing, which is fixed in the plate of the cell. The bearing is of a heavy-duty type and can allow an instrument as heavy as $150 \mathrm{~kg}$ to be mounted at the back. To accommodate the back-end instruments of different focal lengths, the secondary mirror can be moved up $(20 \mathrm{~mm})$ and down $(60 \mathrm{~mm})$ remotely from a console by a stepper motor drive giving a total range of 300 to $2200 \mathrm{~mm}$ from the Cassegrain plate.

The telescope was commissioned in 1995 and since then regular astronomical observations are being made. Observations are being carried out using different observing techniques viz. photometry, imaging, spectroscopy and polarimetry. The back-end instruments that are currently in operation at the Mt. Abu Observatory on a regular basis are as follows: (i) NICMOS Camera System (256 × 256 pixel IR Array) (ii) Two-channel infrared fast-photometer (iii) Imaging Fabry-Perot Spectrometer (iv) Fiber-Linked Astronomical Grating Spectrograph (FLAGS) (v) Optical Photo-Polarimeter and (vi) CCD Camera System. The CCD Camera is used for the variability observations of AGNs, galaxies, stars clusters etc. For the observations of WD J1524 at MIRO we used this CCD camera (Baliyan, Joshi \& Ganesh 2007) details of which are given in the following:

Pixcellent Imaging Ltd, Cambridge EEV CCD 55-30 Grade 0

$1296 \times 1152$ pixels

Pixel size 22.5 microns

Active area $28 \times 26 \mathrm{~mm}$

Read noise 4 electrons rms

Pixel scale 0.48 arcsec/pixel

The CCD is back-end illuminated, LN2 cooled 
The filter wheel attached to the CCD is automated and has 6 slots for UBVRI filters alongwith an open slot. The field of view is about $6^{\prime} \times 5^{\prime} .5$. Usually onchip $2 \times 2$ binning is used for normal operation. The detector is cooled by liquid nitrogen to below -110 degrees to minimize the dark current.

WD J1524-0030

As mentioned earlier, the structure of white dwarfs can be used to put constraints on the prior evolutionary stages of such stars. The detection of pulsation frequencies through precision photometry is used as a tool in this study. Keeping in mind the interesting science such a study of white dwarfs leads to, we at the Mt Abu Observatory also took part in the WET program and during some spare time (about 1.5 hours) WD J1524 was monitored. As these were only preliminary and feasibility-testing observations, these as such do not provide any useful input to the WET program on this source. Nonetheless, the source was observed during May 27-28, 2007 at MIRO in R, V and B filters and corresponding light curves were studied. A typical observed CCD field image is shown in Figure 2.

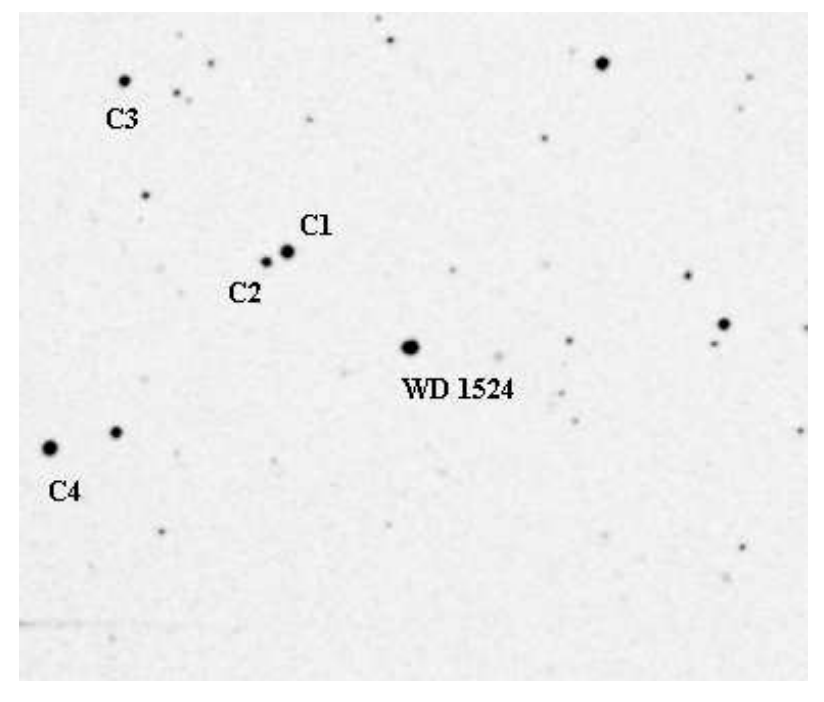

Figure 2: CCD Camera image of the observed field around WD 1524.

The field contains WD J1524-0030 (J2000: 152403.25 -00 30 22.9) and four stars, marked as comparison stars C1, C2, C3 and C4. The exposure time was chosen to be 50 secs in $\mathrm{R}, \mathrm{V}$ and $\mathrm{B}$ filters. Apart from the source 
observations, a large number of bias frames were taken before and after the source observations. Evening twilight sky flats in all the filters used in the observations were also taken. Standard procedures in the IRAF software, such as bias subtraction, flat-fielding and cosmic-rays correction, were followed for data reduction and analysis. On the clean images (bias subtracted, flat fielded), differential photometry was performed using $\mathrm{C} 1$ and $\mathrm{C} 4$ comparison stars along with source WD J1524-0030. Figure 3 shows the temporal variation of the relative magnitudes of WD J1524 with respect to these two comparison stars $\mathrm{C} 1$ and C4. The two differential light curves in Figure 3 are marked as (WD $\mathrm{C} 1$ ) and (WD - C4), respectively. The X-axis shows observation number in the sequence images were taken as a function of time.

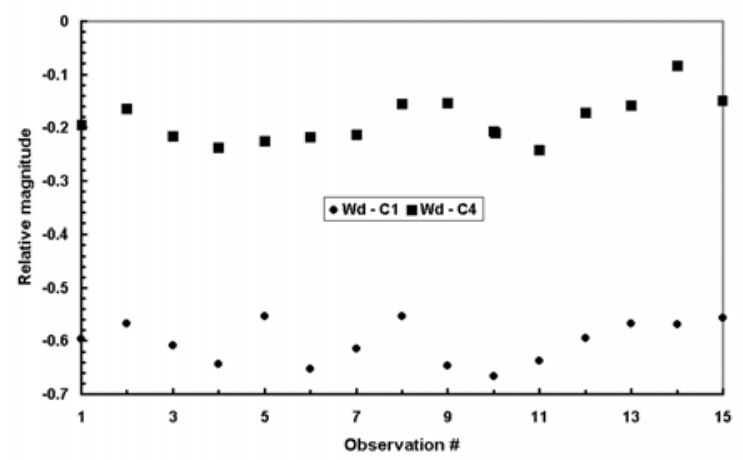

Figure 3: Temporal variations in the observed relative magnitude of WD 1524 with respect to comparison stars $\mathrm{C} 1$ and $\mathrm{C} 4$.

This preliminary photometric data collected for this source was processed using standard correlation and FFT software built in the IDL package. The autocorrelograms for the two series are shown in Figure 4. The Fourier transforms of the two data series of relative magnitudes are shown in Figure 5.

Both the spectra in Figure 5 show a peak at about the same frequency which is equivalent to a periodicity of $210 \mathrm{sec}$. As the data is of very short duration and sampling interval is 70 secs (including readout/overhead time), the resolution is low here and also the confidence limit will be poor. However, since the same periodicity is obtained from the relative magnitudes obtained by the two different comparison stars ( $\mathrm{C} 1$ and $\mathrm{C} 4$ ), it gives some confidence in this estimate. Needless to say that longer monitoring with fast sampling will help attain better estimates of the periodicities in the source.

The present photometry results and the estimated periodicity are indicative that such observations and the procedure adopted can be used to provide vari- 


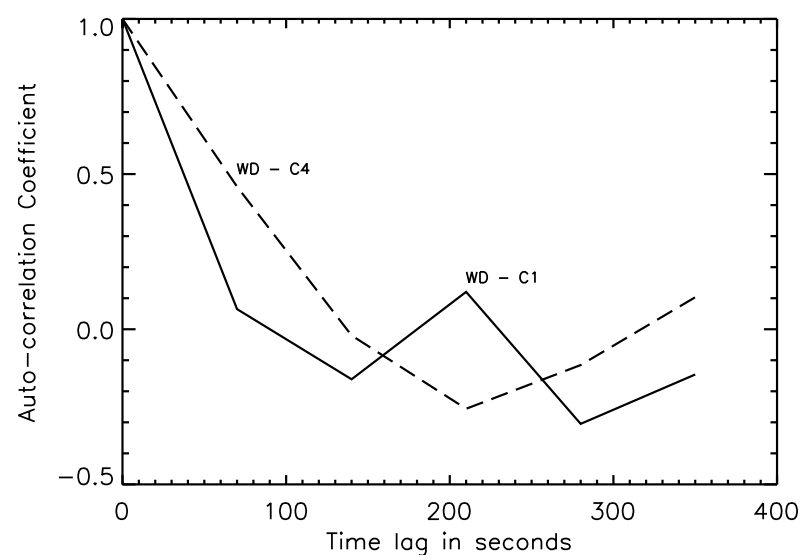

Figure 4: Auto-correlograms of the relative photometric data series.

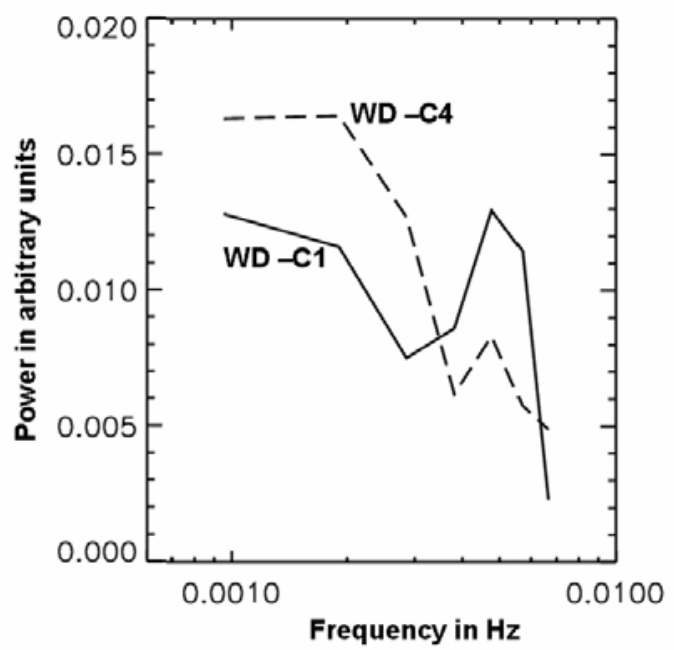

Figure 5: Power spectrum of relative photometric magnitudes.

ability information on WD J1524 and objects of this nature. In future we intend to participate in WET observing program to study pulsating white dwarfs, starting with November 2007 campaign on G38-29. The CCD instrument used in the present observations is not entirely suitable to study pulsating white dwarfs, because it has a substantial dead time (about 14 seconds) between exposures. 
We are in the process of installing a fast CCD (EMCCD) for this type of observations which will enable us much faster sampling (with more than 20 frames per second) and more accurate photometry.

\section{Conclusion}

Though these observations on the WD1524 were in exploratory mode, we could see, from the optical photometry of very limited observation period, variations in the light-curve of the source. The Fourier transform analysis gives a period of about 210 seconds. We feel that present set up can be usefully employed for the WET Campaigns on white dwarfs in future. We also intend to use fast CCD (EMCCD) in future to overcome the large readout time problem.

Acknowledgments. Research at PRL is supported by the Department of Space, Gov. of India. One of us (HOV), thanks the organizers of DARC/WET Workshop 2007 to partially support his participation which provided much required interaction with active workers in the field.

\section{References}

Baliyan, K. S., Joshi, U. C., \& Ganesh, S. 2007, presented at the INCURSI-2007, held at NPL, New Delhi, Feb. 21-24, 2007, AST-4:58

Deshpande, M. R. 1995, BASI, 23, 13

Mukadam, S. A., Montgomery, M. H., Winget, D. E., et al. 2006, ApJ, 640, 956

Winget, D. E. 1998, JPCM, 10, 11247 